\title{
EL PROCESO DE GENERALIZACIÓN MATEMÁTICA: ALGUNAS REFLEXONES EN TORNO A SU VALIDACIÓN
}

\author{
JHONy AlexX́NDER Villa OCHOA*
}

\section{Res:untum}

En la actualidad existen diferentes perspectivas para la iniciación al álgebra escolar, entre ellas se destacan la perspectiva histórica, de solución de problemas, la funcional, de modelación y la de generalización (en cuanto a patrones numéricos y geométricos, y de las leyes que gobiernan las relaciones numéricas). El presente documento intenta plantear algunas reflexiones en torno a esta última perspectiva, la de la generalización, en lo referente a sus procesos de validación. Estas ideas se convierten en un avance de la investigación: "Herramientas para la validación de una generalización matemática", realizada y financiada por el Centro de investigaciones Educativas y Pedagógicas de ASDEM; dichas reflexiones se convierten en un elemento útil en el momento de pensar actividades para introducir el álgebra en la asignatura de matemática.

\section{Paldatora chave}

Generalización matemática, Validación.

* Licenciado en Matemáticas y Física, Universidad de Antioquia, Especialista en Enseñanza de las Matemáticas y Magíster en Educación Matemática de la Universidad de Antioquia. Profesor de Matemáticas de la Universidad de Antioquia y del Instituto Tecnológico Metropolitano. Coordinador Académico del programa de Educación formal para Adultos del ITM y del grupo de investigación "Formación y Didáctica para Adultos" (FORDAD). Integrante del grupo de investigación "Didáctica de las Matemáticas y la Física" de la Universidad de Antioquia reconocido por Colciencias en la convocatoria de 2005, E-mail: javo@epm.net.co 


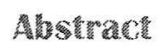

Nowadays exists different perspectives for the algebra school initiation, between them the historical perspective, problem solving, the functional, modeling and generalization (as numeric and geometric patterns, and the laws that govern the numeric relationships) stands out. This document attempt to explain some reflections around this last perspective, the generalization, concerning to their validation processes. These ideas become an investigation advance: "Mathematical generalization and validation Tools", carried out and financed by the ASDEM Educational and Pedagogical Researching Center; this reflections become a useful element in the moment to create activities to introduce the algebra in mathematics's subject.

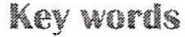

Mathematical Generalization, Validation. 


\section{Wrrtoducción}

La generalización es un proceso al cual algunos investigadores en Educación Matemática han dedicado varios trabajos; Mason $(1999,16)$ afirma que la generalidad es la vida de las matemáticas y que el álgebra es el lenguaje con el que se expresa dicha generalidad; Arzaquiel, (1993) hace una reflexión sobre dicho proceso y los errores que se pueden presentar dentro del mismo; además sugiere cierto tipo de actividades para el trabajo en el aula. Por otro lado Radford (1996) llama la atención sobre los procesos de validez en el proceso de la generalización.

La generalización está presente en muchos contextos, de está forma, en muchas de las situaciones de nuestro diario acontecer se presenta haciendo referencia a eventos donde "generalmente ocurre que..." lo cual es interpretado como "casi siempre ocurre que...". En el contexto de las matemáticas esta misma palabra exige el cumplimiento de la propiedad para todos los elementos de una colección determinada; es decir, para generalizar una regularidad detectada en un caso particular a todo un conjunto, el elemento de éste para el cual se reconoce dicha regularidad ha de ser cualquiera del conjunto. En el campo de la Educación Matemática este término se encuentra como un proceso que involucra el reconocer en diferentes patrones (aritméticos y geométricos), una serie de relaciones de variantes e invariantes entre los diferentes términos y un modo sucinto para expresarlo.

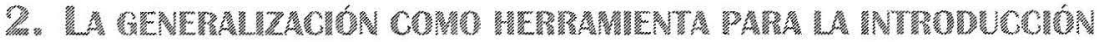 AL. ALLERBRA ESCOLAR}

Muchas veces en el aula de clase no se hace claridad sobre el significado y las diferentes variables que intervienen en el proceso de generalización; con frecuencia se tiende a hacer limitaciones a este proceso, en palabras del grupo Azarquiel (1993: 30):

generalizar no es sólo pasar de una colección de casos particulares a una propiedad común, a una expresión que las englobe ni 
tampoco es definir, a partir de las propiedades de un objeto un campo de objetos caracterizados por cumplirlas (por ejemplo un cuadrilátero tiene dos diagonales, ¿qué figuras tienen sólo dos diagonales?). También se generaliza cuando se transfieren a situaciones propiedades que se cumplen en otras, $y$, en general cuando se amplía el ámbito de definición de una ley.

Mason (1996:67) por su parte afirma:

La generalización es usualmente tomada como una actividad inductivamente empírica en la cual uno acumula muchos ejemplos y detecta el patrón. Pero la generalización más poderosa es usualmente bastante diferente. Hilbert (Courant, 1981) y Davydov (1990) se refieren ambos al hecho de dominar un solo ejemplo que, con un énfasis apropiado y la consecuente ignorancia de rasgos especiales, sirve como un ejemplo genérico en el cual puede ser leído lo general. Eso no es un rasgo distante de los matemáticos avanzados, pero pueden ser experimentados en todos los niveles.

La generalización como cualquier otro proceso sugiere el desarrollo de una serie de habilidades que dan sentido a dicho proceso y en algunos casos se convierten en criterios para categorizar los distintos razonamientos que en él se pueden encontrar. En este orden de ideas Mason (1999) sugiere algunos aspectos que deben tenerse en cuenta en el proceso de la generalización, a saber: a) la visión de la regularidad, la diferencia, la relación (el vero), b) su exposición verbal (decir, expresar) y c) su expresión escrita, de la manera más precisa y sucinta posible (registrar).

'ver' hace relación a la identificación mental de un patrón o una relación (ver un patrón puede ocurrir después de un periodo de tiempo trabajando con un número de ejemplos particulares), y con frecuencia esto sucede cuando se logra la identificación de un algo común, logro que va acompañado de una sensación de regocijo. El 'decir', ya sea a uno mismo o a alguien en particular, es un intento de articular en palabras esto que se ha reconocido. 'Registrar' es hacer visible el lenguaje, lo cual requiere un movimiento hacia los símbolos y la comunicación escrita (incluyendo los dibujos) ;...(Mason, 1999, p 17). 


\section{LA WALDACLON: UND FASE EN EL PROCESO DE GENERALUZACION}

En algunas oportunidades, el proceso de generalización en una situación termina cuando los estudiantes registran lo que han observado en la misma; pocas veces se realiza un proceso de reflexión con aquello que los estudiantes "ven, dicen y registran". De este aspecto surgen entonces una serie de interrogantes entre los cuales cabe destacar: ¿Quién valida el resultado de una generalización en el aula? ¿Cómo se establece la validez del conocimiento matemático en la escuela? En la mayoría de los casos en las aulas de clase se considera válido todo aquello que resulta de aspectos como: la autoridad del docente, la agilidad perceptual de los estudiantes y el cumplimento para unos casos particulares de expresión general, entre otros.

Algunos investigadores se han preocupado por los procesos de validación en la generalización, así por ejemplo Radford (1996) llama la atención argumentando que desde el punto de vista didáctico es preciso tomar en cuenta que la generalización depende de los objetos matemáticos que se estén generalizando y agrega que la generalización no es una actividad libre de contexto pues hay tipos de generalizaciones que pueden ser todos muy diferentes.

Es necesario entonces dedicar tiempo a generar reflexiones en torno a la naturaleza y validación de los procesos de generalización en patrones aritméticos y geométricos [1]; aquí algunas de ellas:

La generalización en patrones geométricos parece tener unas características particulares y significativamente diferentes a la generalización en patrones aritméticos. En primer lugar podemos ver que en los patrones geométricos es posible identificar una serie de "variables visuales" que en los patrones aritméticos por naturaleza misma no existen. Algunas de estas variables son el agrupamiento y la distribución.

Variable "Agrupamiento": cuando se presenta a los estudiantes una secuencia geométrica es imposible predecir una única forma de asociar o agrupar las diferentes unidades del patrón; existen variadas formas de asociar los elementos de un patrón geométri- 
co, cada una de las cuales orientaría el proceso de generalización a alcanzar determinado tipo de expresión simbólica, este tipo de variable se ilustra en el siguiente ejemplo:

Se tienen piscinas de forma hexagonal y se desea cubrir su contorno con baldosas de igual forma como se muestra en la siguiente figura:
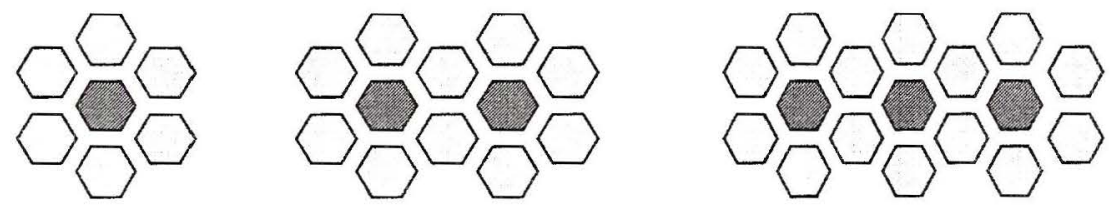

La siguiente tabla recoge los primeros datos de este patrón:

n: $\quad$ Posición

$f(n)$ : Número de baldosas hexagonales alrededor de las piscinas

\begin{tabular}{|l|c|c|c|c|}
\hline$n$ & 1 & 2 & 3 & $\ldots$ \\
\hline$f(n):$ & 6 & 10 & 14 & $\ldots$ \\
\hline
\end{tabular}

Algunas de las diferentes formas de agrupamiento pueden ser: para $n=3$.

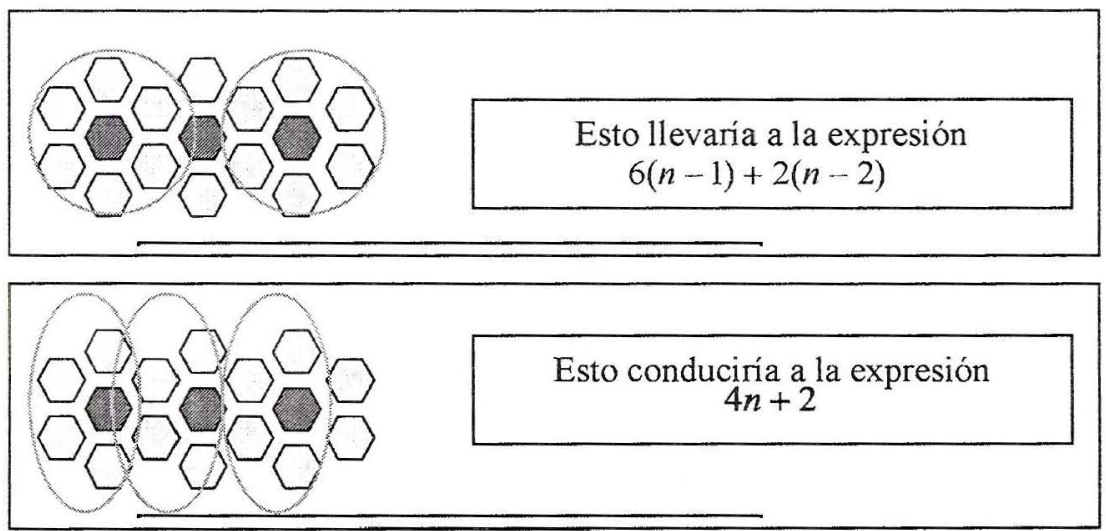



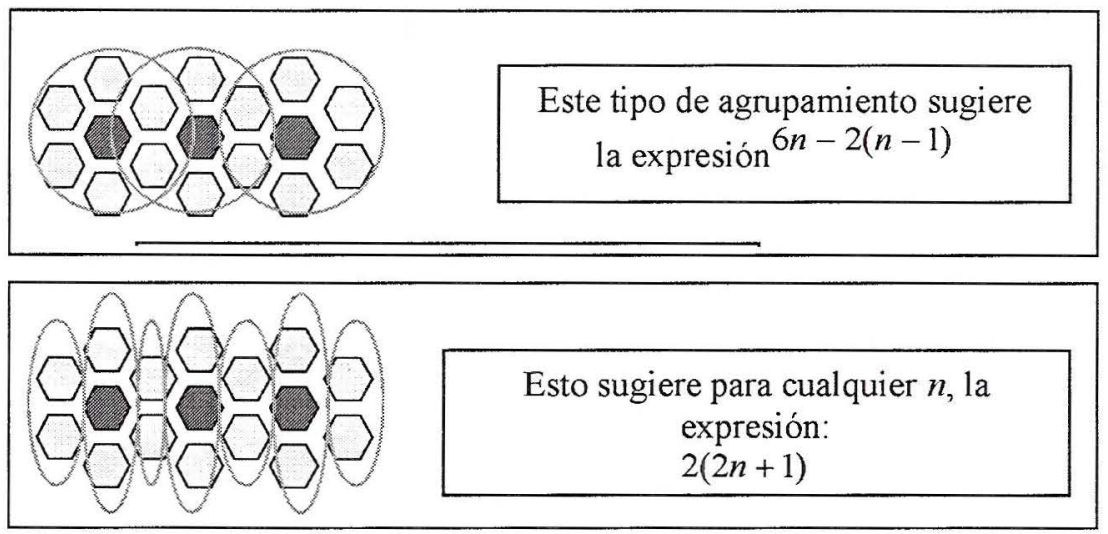

Lo importante de esta variedad de expresiones, es que cada una de ellas refleja una forma diferente de visualización, del reconocimiento de variables e invariantes, de las relaciones entre ellas y por lo tanto una forma diferente de pensar la situación. En el caso de la sucesión anterior, la construcción de la expresión $4 n+2$ implica el reconocimiento de la variación del número de piscinas en términos de los números naturales y el reconocimiento de dos invariantes: la primera es "cuatro baldosas" que se encuentra por cada una de las piscinas; y la segunda es "dos baldosas" que se encuentra en cada miembro de la sucesión, independiente del número de piscinas. La ecuación resulta al reconocer la relación multiplicativa entre la invariante "cuatro baldosas" y la variable "número de piscinas" y su relación aditiva con la invariante "dos baldosas". Cabe aclarar que esta estrategia de generalización implica un buen desarrollo del pensamiento multiplicativo. A pesar de ello, es posible que los estudiantes puedan construir expresiones de manera recursiva a partir de la observación de variables e invariantes y la identificación única de relacionas aditivas entre ellas. En el caso de la sucesión anterior, observar que la primera figura está compuesta de seis baldosas, la segunda está compuesta de las mismas seis de la primera figura más cuatro baldosas adicionales, la tercera figura está conformada por el número de baldosas de la segunda figura aumentada en cuatro baldosas más, y así sucesivamente. Este tipo de razonamiento lleva 
a la expresión recursiva $f(n)=f(n-1)+6$ : que se convierte en un primer nivel de la generalización (base para el estudio posterior de las progresiones aritméticas) pero de igual manera no es lo suficientemente efectiva cuando se trata de calcular el número de baldosas para un término lo suficientemente alto, por ejemplo para $n=50$ ya que implica el conocimiento del término para $n=49$ y este a su vez del término $n=48$ y así sucesivamente.

Es importante notar que todas las expresiones obtenidas en las diferentes formas de agrupación resultan ser equivalentes; este hecho parece ser lo suficientemente convincente para justificar este proceso de generalización, de hecho, Azarquiel (1993:43) afirma que "el término general de una serie de figuras se puede obtener mediante varias expresiones pero que son algebraicamente equivalentes. Precisamente esta equivalencia es la que se puede colocar de manifiesto y servir de demostración en un cierto sentido, al construir distintas expresiones que describen una misma realidad" [2]. La incertidumbre con respecto a la validez en la expresión simbólica, parece resuelta en este patrón por medio de la "variable agrupamiento". Sin embargo es preciso plantearse la pregunta ¿con cualquier tipo de agrupamientos en la secuencia, se obtendrán "siempre" expresiones equivalentes? Una aproximación parcial a esta pregunta apuntaría a una respuesta positiva pues aunque existen muchas más expresiones que satisfacen algunos datos de la secuencia $\frac{n^{5}-15 n^{4}+85 n^{3}-225 n^{2}+754 n+120}{120}$ (i.e. satisface las condiciones del problema para los primeros cinco casos particulares), sin embargo, según Villa (2001) dichas expresiones (polinómicas) tienen un grado igual o superior al número de casos particulares en los cuales se desea que coincidan, lo cual hace poco viable encontrar dichas expresiones por medio de agrupamientos en los elementos de la secuencia.

Variable "Distribución" es común encontrar diferentes tipos de patrones que al tratar de generalizarlos pueden llevar a una misma expresión simbólica; por ejemplo, los dos siguientes patrones pueden obedecer a la expresión $\frac{n(n+1)}{2}$. 


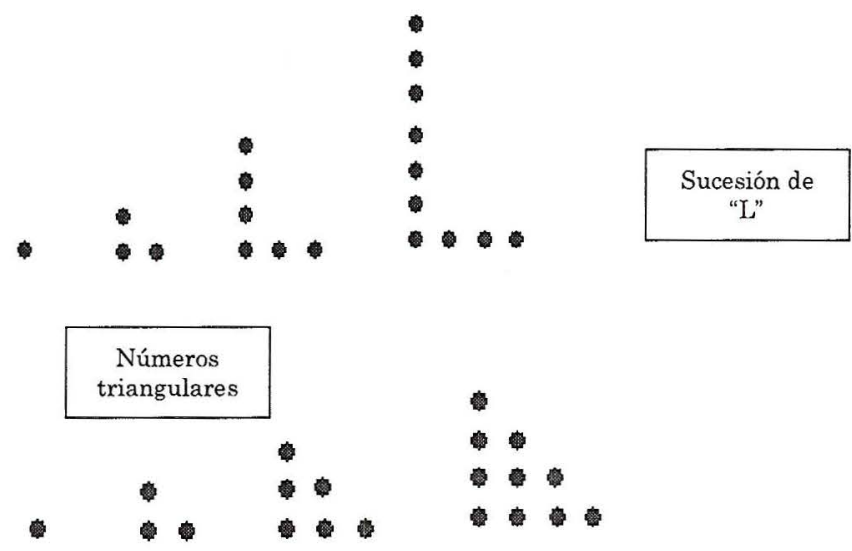

En ambas sucesiones es posible observar que con una reorganización de las piezas de una de las figuras se puede obtener la otra, y de esta forma cualquier expresión general para una de ellas es también una expresión para la otra.

En la generalización de patrones aritméticos, se ponen en juego una serie de habilidades con el trabajo con números, así por ejemplo, dada la sucesión de números $1,3,6,10, \ldots$ es posible que el estudiante observe que "la secuencia obtenida con las diferencias entre dos elementos consecutivos de la sucesión da como resultado la sucesión de números naturales" o que "las segundas diferencias entre dos elementos consecutivos 'siempre' son constantes", pero, ¿son estas observaciones hechos suficientes para garantizar la existencia de una única expresión general? En efecto, el estudiante puede que después de algunos cálculos llegue a que la expresión $\frac{n(n+1)}{2}$ representa estos términos de la sucesión y con esto quede satisfecho. En este caso se hace referencia a Radford (1996: 110) cuando afirma que un procedimiento de generalización g nos lleva a una conclusión $\alpha$, comenzando por una secuencia de "hechos observados", $a_{1}, a_{2}, a_{3}, \ldots, a_{n}$. Podemos escribir esto como: $a_{1}, a_{2}, a_{3}, \ldots$, $a_{n} \rightarrow \alpha$ ( $\alpha$ es derivada de $\left.a_{1}, a_{2}, a_{3}, \ldots, a_{n}\right)$.

Los hechos $a_{1}, a_{2}, a_{3}, \ldots, a_{n}$ son interpretados de acuerdo con una cierta manera de pensamiento, dependiendo del conocimiento y 
los propósitos del observador. Esta forma de pensamiento resulta de la conceptualización de los objetos matemáticos que tiene el observador y las relaciones involucradas en y entre los hechos $a_{1}$, y lleva a una particular forma de pensamiento matemático.

En los patrones aritméticos, al no existix formas visuales que permitan una manipulación de los elementos y que cada nuevo término aparece por un proceso inductivo que depende de la experiencia en el dominio numérico del estudiante, se encuentran múltiples expresiones simbólicas que satisfacen los datos dados en el patrón; de esta forma, la secuencia: $1,3,6,10, \ldots$ puede ser presentada por $\frac{n(n+1)}{2}$; o por las expresiones

$\frac{1}{2520} n^{7}-\frac{28}{2520} n^{6}+\frac{322}{2520} n^{5}-\frac{1960}{2520} n^{4}+\frac{6769}{2520} n^{3}-\frac{11872}{2520} n^{2}+\frac{14328}{2520} n-\frac{5040}{2520} \mathrm{y}$

$\frac{1}{114065280} n^{9}-\frac{628}{114065280} n^{8}+\frac{67122}{114065280} n^{7}-\frac{1595160}{114065280} n^{6}+\frac{17282769}{114065280} n^{5}$ que $-\frac{102074532}{114065280} n^{4}+\frac{346342268}{114065280} n^{3}-\frac{607413200}{114065280} n^{2}+\frac{713456640}{114065280} n-\frac{252000000}{114065280}$

que resultan de solucionar un sistema de ecuaciones a partir del polinomio general $f(n)=a_{k} n^{n}+a_{k-1} n^{k+1}+\ldots+a_{2} n^{2}+a_{1} n+a_{0}$ asignándole valores a $n$ y $f(n)$ según el número de valores que se quieren calcular. De igual manera se pueden obtener a partir de herramientas del Álgebra Lineal y de interpolación por Métodos Numéricos.

Ante la imposibilidad de encontrar una única expresión simbólica que represente una secuencia de números, el proceso de validación debe incluir las competencias de los estudiantes para dar explicaciones y argumentos de los procedimientos elaborados, la justificación de la selección de una estrategia para abordar el problema, la comprobación de la expresión mediante la satisfacción de datos del patrón, y la presencia en los razonamientos de los estudiantes de algunas características de las generalizaciones matemáticas como:

a. Todas incluyen, en forma explícita o implícita, el cuantificador universal. 
b. Poseen variables que están definidas sobre dominios determinados. Estos dominios condicionan el valor de verdad de la generalización

c. Son susceptibles de asociarles un determinado valor de verdad. Establecer y reconocer una justificación; este valor de verdad se llama: "demostrar la generalización".

d. Existen diversas formas y grados para establecer el valor de verdad.

e. Son susceptibles de ser instanciadas escogiendo valores específicos desde los dominios de las variables.

f. Están construidas usando los conceptos como elementos básicos.

g. Existen dos categorías de competencias asociadas a cada generalización. De Tipo I que corresponden a aquellas necesarias o requeridas para poder instanciar adecuadamente la generalización y las de Tipo II que corresponden a las nuevas competencias provenientes de la capacidad de usarla pertinente y exitosamente. (González, en http://lemc.usach.cl/jt/ponencia. doc)

\section{H. CONCUUSIONES}

La perspectiva de la generalización para la iniciación al álgebra es una ruta que tiene grandes ventajas en cuanto al desarrollo de habilidades algebraicas de los estudiantes, sin embargo como afirma Radford (1996: 108) La generalización como una herramienta didáctica no puede evitar el problema de la validez y la validez es, en sí misma, una idea muy compleja. Esto no significa que la generalización no pueda ser usada como un puente útil hacia el álgebra. Yo deseo señalar que el uso de la generalización supone que debemos estar preparados para trabajar con una herramienta (lógica) adicional en el salón de clase.

La generalización de patrones aritméticos y geométricos es de naturaleza diferente y como tal es preciso que se tome conciencia 
de dichas diferencias a la hora de preparar actividades para el aula, dado que en cualquiera de ellas se hacen presentes una serie de variables que no necesariamente hacen presencia en la otra. En la generalización de patrones de carácter geométrico existe cierto grado de certidumbre (sobre la validez) ofrecida por algunas de las variables visuales que se pueden practicar y al igual que en los patrones aritméticos es necesario incluir las competencias de los estudiantes para dar explicaciones y argumentos de los procedimientos elaborados, la justificación de la selección de una estrategia para abordar el problema y la comprobación de la expresión mediante la satisfacción de datos del patrón.

La imposibilidad de encontrar una única expresión simbólica que generalice determinado tipo de patrón aritmético sugiere que en el aula se incluyan reflexiones sobre la variedad de las expresiones y el contexto en el cual surgen.

La validación, entendida como una fase que da al proceso de generalización en el aula de clase cierto grado de certidumbre y seguridad frente a los resultados obtenidos en el proceso, es una tarea que extrapola la común idea positivista de demostración-deducción o la necesidad de encontrar una respuesta única y correcta por parte del alumno hacia el profesor. Es preciso recontextualizar o redefinir el significado, dar un lugar al estudiante para que él mismo haga parte del proceso de validación, y establecer determinadas condiciones de dicho proceso de tal manera que sea posible, realizarlo y así incluirlo como una fase dentro del grande y maravilloso mundo de la generalización.

\section{REFERENCIAS}

[1] Utilizo el término patrones aritméticos y geométricos en lugar de patrones aritmético-geométricos porque considero que ambos tienen características muy diferentes que pueden quedar ocultas al mencionarlas como un solo tipo de patrón.

[2] En esta cita el subrayado lo he hecho para resaltar la idea de demostración en relación con la de validación. 


\section{Pigluocrafia}

GONZÁLEZ, H. Propuestas para la investigación que emergen del estudio de una generalización matemática. Ponencia presentada a la Sociedade Brasileira de Educação Matemática. São Paulo. En: http://lemc.usach. $\mathrm{cl} / \mathrm{jt} /$ ponencia.doc (Consultada 24 noviembre de 2005).

GRUPO AZARQUIEL. Ideas y actividades para trabajar álgebra. Madrid: Síntesis. 1993.

MASON, J.; GRAHAN, A.; PIMM, D. y GOWARD, N. Rutas/raíces hacia el álgebra. Tunja: Universidad Pedagógica y Tecnológica de Colombia, 1999.

MASON, J. Expressing generality and roots of algebra. En: BEDNARZ, N.; KIERAN, C. y LEE, L. (Eds). Approaches to algebra. Perspectives for research and teaching. Dordrecht: Kluwer, 1996; p. 65-86.

RADFORD, L. Some flections on teacheing algebra through generalization. En: BEDNARZ, N.; KIERAN, C. y LEE, L. (Eds). Approaches to algebra. Perspectives for research and teaching. Dordrecht: Kluwer, 1996; p. 107, 114.

VILLA, A. Identificar funciones polinómicas. Una tarea no siempre realizable. En: EMA, Bogotá. Vol. 6, No. 3 (jul. 2001); p. 290-298. 